S. D'Arco, J.A. Suul, and J. Beerten, "Time-invariant state-space model of an ac cable by dqrepresentation of frequency-dependent pi-sections," Proc. IEEE PES ISGT Europe 2019, Bucharest, Romania, 29 Sep 2019-02 Oct 2019, 5 pages.

Digital Object Identifier: 10.1109/ISGTEurope.2019.8905577

URL:

https://ieeexplore.ieee.org/abstract/document/8905577

(C) 2019 IEEE. Personal use of this material is permitted. Permission from IEEE must be obtained for all other users, including reprinting/ republishing this material for advertising or promotional purposes, creating new collective works for resale or redistribution to servers or lists, or reuse of any copyrighted components of this work in other works. 


\section{Time-Invariant State-Space model of an AC Cable by $d q$-representation of Frequency-Dependent $\pi$-sections}

\author{
Salvatore D' Arco $^{1}$ \\ 1 SINTEF Energy Research \\ Trondheim, Norway \\ salvatore.darco@sintef.no
}

\author{
Jon Are Suul ${ }^{1,2}$ \\ 2 Dept. of Engineering Cybernetics \\ NTNU \\ Trondheim, Norway
}

\author{
Jef Beerten ${ }^{3}$ \\ ${ }^{3}$ Dept. of Electrical Engineering \\ KU Leuven - EnergyVille \\ Heverlee - Genk, Belgium
}

\begin{abstract}
This paper introduces an approach for frequencydependent pi-section (FD- $\pi$ ) modelling of ac cables which enables a time-invariant state-space representation that accounts for the frequency-dependent cable characteristics. The proposed approach relies on vector fitting to obtain parametric values for parallel $R L$-branches representing the frequency-dependency of the series impedance of the cable in the stationary frame. The number of cascaded FD- $\pi$ sections with their corresponding shunt capacitance is then selected in order to represent the internal resonance frequencies of the cable up to the required modelling bandwidth. The resulting stationary frame model is transformed to a synchronously rotating dq reference frame to obtain a state-space formulation compatible with the timeinvariant representation of other system components. The presented model allows for accurate time-invariant state-space modelling of power systems with long ac-cables where electromagnetic transients cannot be neglected in small-signal stability studies. The characteristics of the time-invariant cable model is evaluated in the frequency domain, illustrating the impact of the number of $\pi$ sections and parallel $R L$ branches on the accuracy and the associated oscillation modes of the state-space model.
\end{abstract}

Index Terms-Eigenvalue analysis, HVAC cable modelling, vector fitting, small-signal dynamics, state-space modelling

\section{INTRODUCTION}

The utilization of power electronic technology is currently increasing throughout the power system [1]-[3]. Thus, power electronic converters are becoming widely utilized as grid interfaces of loads and distributed generation, as well as for reactive power compensation and HVDC transmission [4][6]. The fast control actions and the harmonic filters of grid connected power electronic converters are introducing new and faster dynamics in the power system. Consequently, emerging modern power systems with significant power electronic conversion capacity leads to new requirements for the models and tools needed in power system stability analysis [7], [8].

This work was supported by the project HVDC Inertia Provision (HVDC Pro), financed by the ENERGIX program of the Research Council of Norway (RCN) with project number 268053/E2, and the industry partners; Statnett, Equinor, RTE and ELIA. The work of Jef Beerten is funded by a grant from the Research Foundation - Flanders (FWO).

978-1-5386-8218-0/19/\$31.00 (c)2019 IEEE
In traditional methods for analysis of large scale power systems, the impedances of lines and cables are usually modelled by algebraic phasor representation [9]. However, such phasorbased models, cannot represent the electromagnetic transients in the network and the response to fast control actions of power electronic converters. Thus, hybrid modelling approaches have been proposed, where algebraic models are utilized for parts of the network while electromagnetic transient models are utilize to represent the network close to dominant power electronic converters [10].

For eigenvalue-based small-signal stability analysis of power systems, a time invariant model is necessary, implying that all variables should converge to an equilibrium point during stable steady-state operation [9]. Thus, modelling of the electromagnetic dynamics in transmission systems requires that the state equations representing the system dynamics account for the periodicity in the ac signals. This is typically achieved by expressing the model by $d q$ variables in a synchronously rotating reference frame (SRRF). Several recent studies have evaluated the need for representing the electromagnetic dynamics of transmission systems in smallsignal stability studies [11]-[14]. However, these studies usually assume a lumped parameter representation of transmission lines or cables. Thus, the $d q$ frame state equations of a lumped series impedance are usually introduced for representing the network dynamics [7], [8]. Alternatively, a traditional $\pi$ section model, [15], [16] or a series of $T$-section models with fixed parameters [17], [18] can be represented by state equations in the $d q$ reference frame.

For studies of HVDC systems, it has recently been demonstrated that the use of classical cascaded $\pi$-sections for representing dc cables can give rise to a significant underestimation of the internal damping effects caused by the frequencydependent characteristics of the cable [19]. Therefore, utilization of classical $\pi$ section models in small-signal statespace analysis of HVDC transmission schemes can result in identification of poorly damped modes or even false prediction of instabilities that do not represent the actual dynamics of the system. To address this issue, a modelling approach based on frequency dependent $\pi$ (FD- $\pi$ ) sections with mul- 


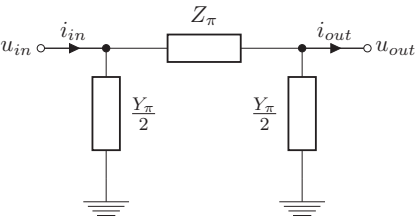

(a)

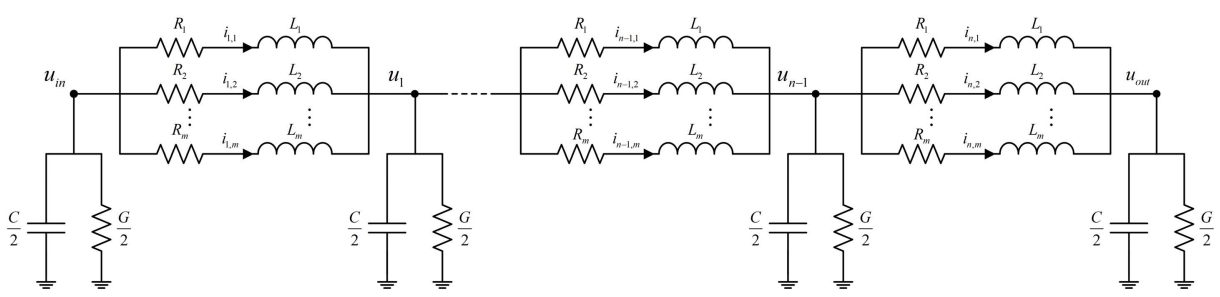

(b)

Fig. 1. Cable modelling by a) $\pi$ representation with frequency dependent impedances; b) circuital representation of the FD- $\pi$ model.

tiple parallel $R L$ branches for accurately representing the frequency-dependent damping effects has been proposed [19][21]. However, this modelling approach has until now been applied only to HVDC cables.

In this paper an approach for FD- $\pi$ modelling of high voltage ac (HVAC) cables is introduced. For simplicity, HVAC transmission by three single-core cables is assumed, allowing for direct application of an FD- $\pi$ model for each phase. Thus, the stationary three-phase FD- $\pi$ model representation can be transformed to a SRRF for obtaining a time-invariant model that accounts for the frequency-dependent damping characteristics of the cable. The validity and accuracy of the proposed modelling approach is demonstrated in the frequency domain. Examples of eigenvalue analysis of a single cable are also presented to demonstrate how the configuration of the FD$\pi$ model in terms of number of sections and number of parallel $R L$ branches in each section influences the representation of the cable dynamics.

\section{FD- $\pi$ MODEL FOR A SINGLE-CORE CABLE}

General considerations on modelling of a single-core cable is presented in this section, as a background for the proposed representation of three-phase ac cables by dynamic $d q$-models.

\section{A. Distributed parameter model of a single-core cable}

The behavior of a single-core cable can be represented by the equivalent circuit in Fig. 1a. The frequency-dependency of the parameters and the influence of the cable length on the impedance due to the distributed parameter characteristics are represented in the series and shunt elements, $Z_{\pi}(s)$ and $Y_{\pi}(s)$. In the Laplace domain, these circuit elements are expressed by [22], [23]:

$$
\begin{gathered}
Z_{\pi}(s)=z(s) \ell \frac{\sinh \gamma(s) \ell}{\gamma(s) \ell}, Y_{\pi}(s)=y(s) \ell \frac{\tanh \frac{\gamma(s) \ell}{2}}{\frac{\gamma(s) \ell}{2}} \\
\gamma(s)=\sqrt{z(s) y(s)}
\end{gathered}
$$

where $z(s)$ and $y(s)$ are the cable impedance and admittance per unit length. These characteristics also define the propagation constant $\gamma(s)$, while $\ell$ denotes the cable length. The series impedance $z(s)$ is assumed to be represented on the form of:

$$
z(s)=r(s)+s \cdot l(s)
$$

where the resistance $r(s)$ and inductance $l(s)$ per unit length are frequency-dependent. Since $z(s)$ is a smooth function in the frequency domain, it is easier to fit with a rational approximation than the function $Z_{\pi}(s)$. The admittance $y(s)$ can be expressed as:

$$
y(s)=g+s \cdot c
$$

In the frequency range of interest for small-signal eigenvaluebased analysis of power systems, the conductance $g$ and the capacitance $c$ per unit length can be assumed constant [24].

The admittance of the cable terminated on a load impedance $Z_{l}(s)$ can be derived from Fig. 1a and (1), which leads to:

$$
Y_{c}(s)=\frac{\gamma(s) \operatorname{coth}(\gamma(s) l)+y(s) Z_{l}(s)}{\gamma(s) Z_{l}(s) \operatorname{coth}(\gamma(s) l)+z(s)}
$$

Thus, the behavior of the cable in the Laplace domain can be represented by a frequency-dependent admittance. The representation of the cable with $Y_{c}(s)$ can be utilized as a reference model for the cable in the frequency domain.

\section{B. $F D-\pi$ model for a single-core cable}

The cable model from Fig. 1a and (5) cannot be expressed in state-space form. Although the impact of the hyperbolic functions are commonly represented by multiple $\pi$-sections for obtaining a rational approximation, such conventional models do not represent the frequency-dependency of the series impedance. For the purpose of state-space modelling and eigenvalue analysis of HVDC transmission systems, a Frequency Dependent $\pi$ (FD- $\pi$ ) model of an HVDC cable was introduced in [19], [20]. As shown in Fig. 1b this model utilizes multiple parallel $R L$ branches to account for the frequency-dependency of $z$. The parameters of the parallel $R L$ branches are obtained to approximate $z$ as:

$$
\frac{1}{z(s)} \approx \sum_{i=1}^{m} \frac{1}{r_{i}+s l_{i}}
$$

by applying vector fitting [20], [23], [25]. As long as the shunt conductance can be assumed constant for the considered frequency range, the parameters for each section of the FD- $\pi$ model can be determined directly from the length of the cable, the selected number of $\pi$ sections, $n$, and the selected number of parallel branches, $m$, for each section. 


\section{FD- $\pi$ MODEL OF AC CABLES}

For simplicity, three phase high voltage ac transmission by three identical and decoupled single-core cables is assumed in the following. Thus, each single-core cables can be modelled according to the approaches presented in section II. For balanced three phase conditions, the $a b c$ phase representation can be reduced to a an orthogonal stationary frame $\alpha \beta$ model. By applying the Park Transformation, the representation of the cable can be further expressed by SRRF $d q$ variables.

With reference to the first section in the model represented in Fig. 1b, the relation between the voltage across the $\pi$ section and the current flowing in the first parallel branch can be expressed in the Laplace domain as:

$$
u_{\text {in }}-u_{1}=\left(R_{1}+s L_{1}\right) i_{1,1}
$$

Thus, the state equation for the current is given by:

$$
s i_{1,1}=-\frac{R_{1}}{L_{1}} i_{1,1}-\frac{1}{L_{1}}\left(u_{i n}-u_{1}\right)
$$

Assuming identical impedance characteristics and no coupling between the phases, the three-phase system can be modelled in a two-phase stationary $\alpha \beta$ reference frame as:

$$
\vec{u}_{i n, \alpha \beta}-\vec{u}_{1, \alpha \beta}=\left(R_{1}+s L_{1}\right) \vec{i}_{1,1, \alpha \beta}
$$

where $\vec{x}_{\alpha \beta}=x_{\alpha}+j x_{\beta}$.

The stationary frame equation in (9) can be transformed to the SRRF defined by the fundamental frequency $\omega$. This transformation is equivalent to replacing the Laplace operator $s$ by $s+j \omega$, leading to:

$$
\vec{u}_{i n, d q}-\vec{u}_{1, d q}=\left(R_{1}+(s+j \omega) L_{1}\right) \vec{i}_{1,1, d q}
$$

where $\vec{x}_{d q}=x_{d}+j x_{q}$.

The state-equations for the $d-$ and $q$-axis current components, can then be obtained from the real and imaginary parts of ((10)), yielding:

$$
\begin{aligned}
& s i_{1,1, d}=-\frac{R_{1}}{L_{1}} i_{1,1, d}-\frac{1}{L_{1}}\left(u_{i n, d}-u_{1, d}\right)+\omega i_{1,1, q} \\
& s i_{1,1, q}=-\frac{R_{1}}{L_{1}} i_{1,1, q}-\frac{1}{L_{1}}\left(u_{i n, q}-u_{1, q}\right)-\omega i_{1,1, d}
\end{aligned}
$$

The $d$ - and $q$-axis state equations in (11) and (12) highlight how the states of the currents in a stationary reference frame FD- $\pi$ model can be transformed into the SRRF. Similar transformations apply also to the states of the voltages associated with the capacitances of the FD- $\pi$ model.

\section{A. State space formulation}

In [19], a state space formulation for the FD- $\pi$ model of a single-core cable is derived on the general form of:

$$
\begin{aligned}
& \dot{x}=A x+B u \\
& y=C x
\end{aligned}
$$

with the states and inputs defined by:

$$
\begin{aligned}
& \boldsymbol{x}=\left[i_{1,1} \ldots i_{1, n} \ldots u_{1} \ldots u_{m-1} \ldots i_{m, 1} \ldots i_{m, n}\right] \\
& \boldsymbol{y} \quad=\quad\left[\begin{array}{ll}
i_{\text {in }} & i_{\text {out }}
\end{array}\right]^{T}
\end{aligned}
$$

The expression of the $\boldsymbol{A}$ and $\boldsymbol{B}$ matrices are not reported here for brevity.

From the general FD- $\pi$ model of a single-core cable on the form given by (13)-(16), a balanced three-phase system can be represented on state-space form in $\alpha \beta$ coordinates as:

$$
\begin{aligned}
& \dot{\boldsymbol{x}}_{\alpha \beta}=\boldsymbol{A}_{\alpha \beta} \boldsymbol{x}_{\alpha \beta}+\boldsymbol{B}_{\alpha \beta} \boldsymbol{u}_{\alpha \beta} \\
& \boldsymbol{A}_{\alpha \beta}=\left[\begin{array}{cc}
\boldsymbol{A} & 0 \\
0 & \boldsymbol{A}
\end{array}\right] \\
& \boldsymbol{B}_{\alpha \beta}=\left[\begin{array}{ll}
\boldsymbol{B} & 0 \\
0 & \boldsymbol{B}
\end{array}\right] \\
& \boldsymbol{C}_{\alpha \beta}=\left[\begin{array}{cc}
\boldsymbol{C} & 0 \\
0 & \boldsymbol{C}
\end{array}\right]
\end{aligned}
$$

where: $\boldsymbol{x}_{\alpha \beta}=\left[\begin{array}{ll}x_{\alpha} & x_{\beta}\end{array}\right]^{T}$. This model is applicable under the assumption that the impedance parameters, the currents and voltages of two axes are balanced and can be expressed without coupling between the phases.

Finally, the stationary fram $\alpha \beta$ model can be transformed into the $d q$ frame. Expanding the resulting equations in the same way as shown in (11) and (12) the time-invariant statespace model can be expressed as:

$$
\begin{aligned}
& \dot{\boldsymbol{x}}_{d q}=\boldsymbol{A}_{d q} \boldsymbol{x}_{d q}+\boldsymbol{B}_{d q} \boldsymbol{u}_{d q} \\
& \boldsymbol{A}_{d q}=\left[\begin{array}{cc}
\boldsymbol{A} & \omega \boldsymbol{I} \\
-\omega \boldsymbol{I} & \boldsymbol{A}
\end{array}\right] \\
& \boldsymbol{B}_{d q}=\boldsymbol{B}_{\alpha \beta} \\
& \mathrm{C}_{d q}=\mathrm{C}_{\alpha \beta}
\end{aligned}
$$

where: $\boldsymbol{x}_{d q}=\left[\begin{array}{ll}x_{d} & x_{q}\end{array}\right]^{T}$.

\section{ANALYSIS AND VERIFICATION OF THE PROPOSED MODEL}

For validating the time-invariant state-space model from (21) and its ability to capture the frequency-dependent characteristics of an HVAC cables, numerical examples of frequency domain analysis are presented for a generic $100 \mathrm{~km}$ ac cable section. Moreover, the effect of the configuration of the proposed $\mathrm{FD}-\pi$ on the transfer functions and on the eigenvalues is presented to highlight the differences from a conventional $\pi$ model.

\section{A. Validation of the FD- $\pi$ model in frequency domain}

Under the assumptions applied in section III, the admittance of a three phase cable in the $d q$ frame can be represented in the frequency domain as:

$$
\left[\begin{array}{c}
i_{d}(s) \\
i_{q}(s)
\end{array}\right]=\left[\begin{array}{cc}
Y_{c, d d}(s) & Y_{c, q d}(s) \\
-Y_{c, q d}(s) & Y_{c, d d}(s)
\end{array}\right]\left[\begin{array}{l}
v_{d}(s) \\
v_{q}(s)
\end{array}\right]
$$




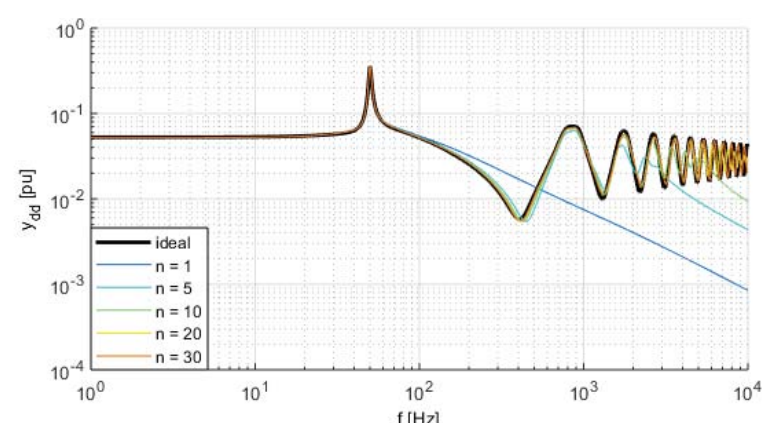

(a)

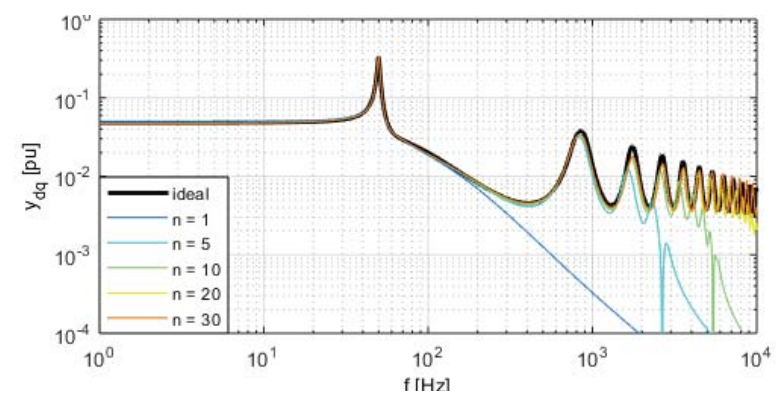

(b)

Fig. 2. Frequency characteristics of admittance components for different number of sections $n$, (with $m=4$ ).

The two transfer functions $Y_{c, d d}(s)=Y_{c, q q}(s)$ and $Y_{c, q d}(s)=-Y_{c, d q}(s)$ can be obtained analytically from the admittance $Y_{c}(s)$ of the single-core cable by a frequency transformation according to the procedure described in [26]:

$$
\begin{aligned}
& Y_{c, d d}(s)=\frac{1}{2}\left(Y_{c}\left(s+j \omega_{0}\right)+Y_{c}\left(s-j \omega_{0}\right)\right) \\
& Y_{c, q d}(s)=\frac{1}{2} j\left(Y_{c}\left(s+j \omega_{0}\right)-Y_{c}\left(s-j \omega_{0}\right)\right)
\end{aligned}
$$

The two expressions in (27) and (28) can be assumed as a reference for the cable behavior in the frequency domain. Thus, the accuracy of FD- $\pi$ models are assessed in the frequency domain by comparison to (27) and (28).

The frequency characteristics of FD- $\pi$ models in terms of $y_{d d}$ and $y_{d q}$ for $m=4$ and different values of $n$ are shown in Fig. 2. The reference models from (27) and (28) are labelled as "ideal" in the figure. By contrast, the frequency characteristics for $n=20$ and different values of $m$ are shown in Fig. 3 and again compared with the reference model. These results clearly indicate that the accuracy of the frequency characteristics increases by considering a model with higher order. In particular, Fig. 2 shows that the effect of increasing the number of $\pi$ sections $n$ is increasing the frequency range where the accuracy can be maintained. Indeed, all the models present a roll-off at high frequencies, but higher values of $n$ are extending the frequency range where the model is valid. Even more obvious is the influence of the number of parallel branches $m$ on the frequency characteristics. Indeed, for the lowest values of $m$ (i.e. $m=1$ and $m=2$ ) the model tends to exhibit wrong resonances both in terms of frequency and

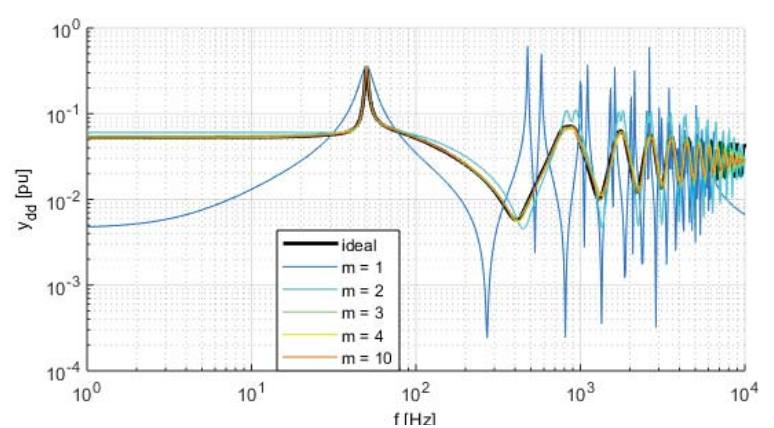

(a)

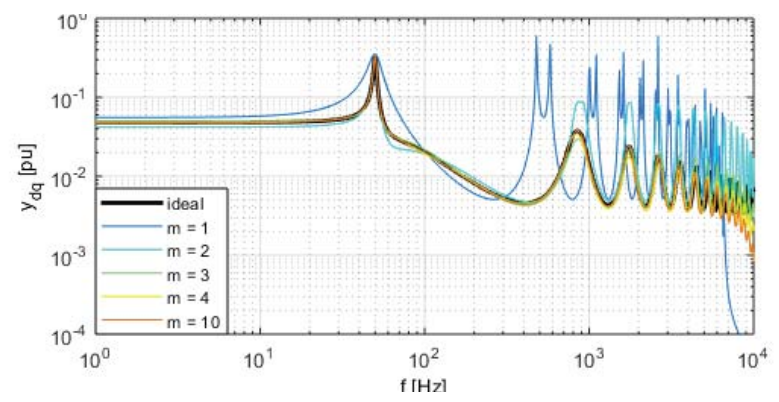

(b)

Fig. 3. Frequency characteristics of admittance components for different number of parallel branches $m$, (with $n=20$ ).

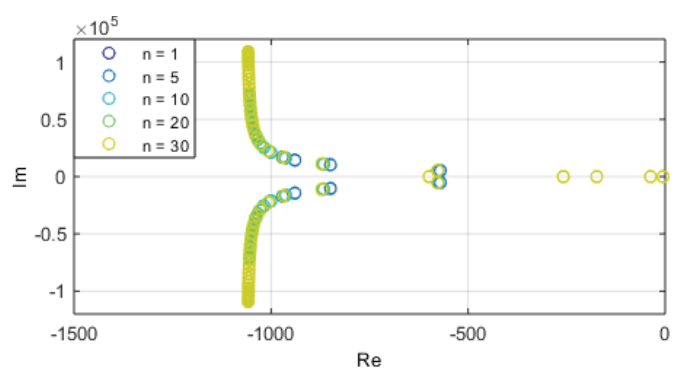

Fig. 4. Comparison of eigenvalues for FD-pi models with different number of sections $n$, (for $m=4$ ).

damping. However, already with $m=3$ this effect tends to disappear. This further confirms that the conventional $\pi$ model corresponding to $m=1$ could be quite inaccurate for eigenvalue-based stability analysis and could be misleading by introducing modes with significant lower damping than a real cable will exhibit. However, it should be noted that the presented results are obtained by enforcing the dc-resistance of the cable for the vector fitting. Thus, a different distribution of the deviations from the reference model would occur if the impedance at the grid frequency would be imposed in the fitting process while relaxing the fitting of the dc-resistance.

\section{$B$. Eigenvalue analysis of dq-frame FD- $\pi$ models}

The impact of $n$ and $m$ on the cable model behavior can also be observed in eigenvalue analysis. The slowest eigenvalues of FD- $\pi$ models with different values of $n$ are shown in Fig. 4 (i.e. eigenvalues with real part below -1500 are not displayed). The eigenvalues tend to align on an almost vertical line, but 


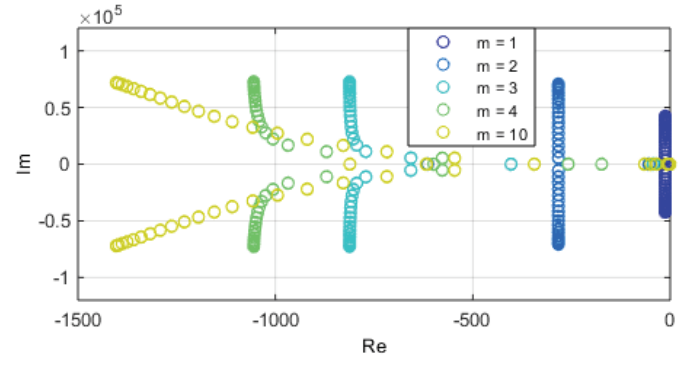

Fig. 5. Comparison of eigenvalues for FD-pi models with different number of parallel branches $m$, (for $n=10$ ).

higher order models extend the range of resonance frequencies captured by the model (i.e. by poles with higher imaginary parts). This corresponds to the observations in the frequency domain, where higher values of $n$ will extend the frequency range captured by the model but do not have a major role in defining the damping. The impact on the eigenvalues of FD- $\pi$ models with different values of $m$ is shown in Fig. 5. In this second case, the eigenvalues clearly present a very different behavior in terms of damping since they tend to align with different slopes in the complex plane. It should be noted that the damping can have a very critical effect on stability and that the value of $m$ should be sufficiently high to avoid false poorly damped modes that could potentially interact with the controls.

\section{CONCLUSION}

This paper introduces a frequency-dependent $\pi$ (FD- $\pi$ ) model of ac cables, intended for eigenvalue-based analysis of small-signal dynamics in power systems. The model extends the concept of the FD- $\pi$ models previously introduced for $\mathrm{dc}$ cables and allows a representation of ac cable dynamics in a rotating $d q$ frame, which is necessary to obtain a time-invariant state-space model for eigenvalue assessment of three-phase ac systems. Analysis in the frequency domain demonstrate how increasing the number $\pi$-sections and parallel $R L$-branches of the FD- $\pi$-model can provide higher accuracy and effectively extend the frequency range where the model can be applied. Moreover, the paper demonstrates that the conventional $\pi$ model with a single parallel branch can lead to significant errors in the damping of internal oscillation modes.

\section{ACKNOWLEDGMENT}

Bjørn Gustavsen at SINTEF Energy Research is acknowledged for his support with the vector fitting tool.

\section{REFERENCES}

[1] D. Boroyevich, I. Cvetkovic, R. Burgos, and D. Dong, "Intergrid: A future electronic energy network?" IEEE Journal of Emerging and Selected Topics in Power Electronics, vol. 1, no. 3, pp. 127-138, 2013.

[2] (2019) Migrate eu project - massive integration of power electronic devices. [Online]. Available: https://www.h2020-migrate.eu/

[3] G. Denis, T. Prevost, M.-S. Debry, F. Xavier, X. Guillaud, and A. Menze, "The migrate project: the challenges of operating a transmission grid with only inverter-based generation. a grid-forming control improvement with transient current-limiting control," IET Renewable Power Generation, vol. 12, no. 5, pp. 523-529, 2017
[4] F. Blaabjerg, A. Consoli, J. A. Ferreira, and J. D. van Wyk, "The future of electronic power processing and conversion," IEEE Transactions on Industry Applications, vol. 41, no. 1, pp. 3-8, 2005.

[5] J. G. Kassakian and T. M. Jahns, "Evolving and emerging applications of power electronics in systems," IEEE Journal of Emerging and Selected Topics in Power Electronics, vol. 1, no. 2, pp. 47-58, 2013.

[6] B. Kroposki, B. Johnson, Y. Zhang, V. Gevorgian, P. Denholm, B.-M. Hodge, and B. Hannegan, "Achieving a 100\% renewable grid: Operating electric power systems with extremely high levels of variable renewable energy," IEEE Power and Energy Mag., vol. 15, no. 2, pp. 61-73, 2017.

[7] Y. Wang, X. Wang, Z. Chen, and F. Blaabjerg, "Small-signal stability analysis of inverter-fed power systems using component connection method," IEEE Trans. Smart Grid, vol. 9, no. 5, pp. 5301-5310, 2018.

[8] M. Raza, E. Prieto-Araujo, and O. Gomis-Bellmunt, "Small-signal stability analysis of offshore ac network having multiple vsc-hvdc systems," IEEE Transactions on Power Delivery, vol. 33, no. 2, pp. 830-839, 2018.

[9] P. Kundur, N. J. Balu, and M. G. Lauby, Power system stability and control. McGraw-hill New York, 1994, vol. 7.

[10] C. Karawita and U. D. Annakkage, "A hybrid network model for small signal stability analysis of power systems," IEEE Transactions on power systems, vol. 25, no. 1, pp. 443-451, February 2010.

[11] Y. Levron and J. Belikov, "Modeling power networks using dynamic phasors in the dq0 reference frame," Electric Power Systems Research, vol. 144, pp. 233-242, 2017.

[12] J. Belikov and Y. Levron, "Comparison of time-varying phasor and dq0 dynamic models for large transmission networks," International Journal of Electrical Power \& Energy Systems, vol. 93, pp. 65-74, 2017.

[13] _ " "Uses and misuses of quasi-static time-varying phasor models in power systems," IEEE Trans. Power Del., vol. 33, no. 6, pp. 3263-3266, 2018

[14] D. Baimel, J. Belikov, J. M. Guerrero, and Y. Levron, "Dynamic modeling of networks, microgrids, and renewable sources in the dq0 reference frame: A survey," IEEE Access, vol. 5, pp. 21323-21335, 2017.

[15] S. Jiang, S. Zhang, X. Lu, B. Ge, and F. Z. Peng, "Resonance issues and active damping in hvac grid-connected offshore wind farm," in 2013 IEEE Energy Conv. Congress and Expo. IEEE, 2013, pp. 210-215.

[16] S. D'Arco and J. A. Suul, "Small-signal analysis of an isolated power system controlled by a virtual synchronous machine," in 2016 IEEE International Power Electronics and Motion Control Conference (PEMC). IEEE, 2016, pp. 462-469.

[17] J. Belikov and Y. Levron, "Integration of long transmission lines in largescale dq0 dynamic models," Electrical Engineering, vol. 100, no. 2, pp. 1219-1228, 2018.

[18] _ _ "A sparse minimal-order dynamic model of power networks based on dq0 signals," IEEE Transactions on Power Systems, vol. 33, no. 1, pp. 1059-1067, 2018.

[19] J. Beerten, S. D'Arco, and J. A. Suul, "Frequency-dependent cable modelling for small-signal stability analysis of VSC-HVDC systems," IET Gener., Transm. Distrib., vol. 10, no. 6, pp. 1370-1381, 2016.

[20] _ "Cable model order reduction for HVDC systems interoperability analysis," in IETACDC 2015, Birmingham, UK, Feb., 10-12 2015, 10 p.

[21] _ , "Identification and small-signal analysis of interaction modes in vsc mtdc systems," IEEE Trans. Power Del., vol. 31, no. 2, pp. 888-897, Apr. 2016.

[22] J. A. Rocendo Macias, A. Gomez Exposito, and A. Bachiller Soler, "A comparison of techniques for state-space transient analysis of transmission lines," IEEE Trans. Power Del., vol. 20, no. 2, pp. 894-903, Apr. 2005.

[23] S. Grivet-Talocia and B. Gustavsen, Passive Macromodelling, Theory and Applications. Wiley, 2016, ch. 12, pp. 563-661.

[24] B. Gustavsen, T. Noda, J. Naredo, F. Uribe, and J. Martinez-Velasco, Power System Transients: Parameter Determination. Taylor and Francis Group, 2010, ch. 3, pp. 137-175.

[25] B. Gustavsen and A. Semlyen, "Rational approximation of frequency domain responses by vector fitting," IEEE Trans. Power Del., vol. 14 no. 3, pp. 1052-1061, Jul. 1999.

[26] D. N. Zmood, D. G. Holmes, and G. Bode, "Frequency domain analysis of three phase linear current regulators," in Conference Record of the 1999 IEEE Industry Applications Conference. Thirty-Forth IAS Annual Meeting (Cat. No. 99CH36370), vol. 2. IEEE, 1999, pp. 818-825. 